\title{
Implementation of a Pragmatic Biomarker-Driven Algorithm to Guide Antibiotic Use in the Pediatric Intensive Care Unit: the Optimizing Antibiotic Strategies in Sepsis (OASIS) II Study
}

Kevin J. Downes, ${ }^{1,2,3,6,9}$ Julie C. Fitzgerald ${ }^{4,7}$ Emily Schriver, ${ }^{2,3}$ Craig L. K. Boge ${ }^{2,3}$ Michael E. Russo, ${ }^{1,6}$ Scott L. Weiss ${ }^{4,7}$ Fran Balamuth, ${ }^{2,5,6}$ Sherri E. Kubis ${ }^{4}$ Pam Tolomeo, ${ }^{8}$ Warren B. Bilker, ${ }^{8}$ Jennifer H. Han, ${ }^{8,9}$ Ebbing Lautenbach ${ }^{8,9}$ Susan E. Coffin, ${ }^{1,2,3,6}$ Jeffrey S. Gerber, ${ }^{1,2,3,6,8}$; for the CDC Prevention Epicenters Program

'Division of Infectious Diseases; ${ }^{2}$ Center for Pediatric Clinical Effectiveness; ${ }^{3 P e d i a t r i c ~ I n f e c t i o u s ~ D i s e a s e s ~ E p i d e m i o l o g y ~ a n d ~ A n t i m i c r o b i a l ~ S t e w a r d s h i p ~ R e s e a r c h ~ G r o u p ; ~}$ ${ }^{4}$ Department of Anesthesiology and Critical Care Medicine; ${ }^{5}$ Division of Emergency Medicine, Children's Hospital of Philadelphia, Philadelphia, Pennsylvania; ${ }^{6}$ Department of Pediatrics; ${ }^{7}$ Department of Anesthesiology and Critical Care Medicine; ${ }^{8}$ Center for Clinical Epidemiology and Biostatistics, Department of Biostatistics, Epidemiology, and Informatics; and the 9Division of Infectious Diseases, Department of Medicine, Perelman School of Medicine, University of Pennsylvania, Philadelphia, Pennsylvania

Background. Biomarkers can facilitate safe antibiotic discontinuation in critically ill patients without bacterial infection.

Methods. We tested the ability of a biomarker-based algorithm to reduce excess antibiotic administration in patients with systemic inflammatory response syndrome (SIRS) without bacterial infections (uninfected) in our pediatric intensive care unit (PICU). The algorithm suggested that PICU clinicians stop antibiotics if (1) C-reactive protein $<4 \mathrm{mg} / \mathrm{dL}$ and procalcitonin $<1 \mathrm{ng} / \mathrm{mL}$ at SIRS onset and (2) no evidence of bacterial infection by exam/testing by 48 hours. We evaluated excess broad-spectrum antibiotic use, defined as administration on days 3-9 after SIRS onset in uninfected children. Incidence rate ratios (IRRs) compared unadjusted excess length of therapy (LOT) in the 34 months before (Period 1) and 12 months after (Period 2) implementation of this algorithm, stratified by biomarker values. Segmented linear regression evaluated excess LOT among all uninfected episodes over time and between the periods.

Results. We identified 457 eligible SIRS episodes without bacterial infection, 333 in Period 1 and 124 in Period 2. When both biomarkers were below the algorithm's cut-points $(\mathrm{n}=48$ Period $1, \mathrm{n}=31$ Period 2), unadjusted excess LOT was lower in Period 2 (IRR, 0.53; 95\% confidence interval, 0.30-0.93). Among all 457 uninfected episodes, there were no significant differences in LOT (coefficient $0.9, P=.99$ ) between the periods on segmented regression.

Conclusions. Implementation of a biomarker-based algorithm did not decrease overall antibiotic exposure among all uninfected patients in our PICU, although exposures were reduced in the subset of SIRS episodes where biomarkers were low.

Keywords. antimicrobial stewardship; biomarkers; critical care; pediatrics.

Prompt initiation of antibiotics in patients with sepsis has been associated with reduced morbidity and mortality $[1,2]$. Broadspectrum agents are necessary initially to maximize the likelihood of providing coverage for potential bacterial pathogens. Yet, many critically ill children who present with systemic inflammatory response syndrome (SIRS) ultimately do not have bacterial infections. Overuse of broad-spectrum antibiotics can lead to patient harm, development of antibiotic resistance, and excess healthcare costs [3]. Given the extent of antibiotic prescribing in pediatric intensive care units (PICUs) $[4,5]$,

Received 27 July 2018; editorial decision 16 October 2018; accepted 18 October 2018; Published online November 22, 2018.

Correspondence: K. J. Downes, MD, Division of Infectious Diseases, Children's Hospital of Philadelphia, 2716 South Street, Suite 10360, Philadelphia, PA 19146 (downeskj@email.chop.edu). Journal of the Pediatric Infectious Diseases Society 2020;9(1):36-43

(C) The Author(s) 2018. Published by Oxford University Press on behalf of The Journal of the Pediatric Infectious Diseases Society. All rights reserved. For permissions, please e-mail: journals.permissions@oup.com.

DOI: 10.1093/jpids/piy113 practical approaches are needed to safely limit broad-spectrum antibiotic use.

Biomarkers such as procalcitonin (PCT) and C-reactive protein (CRP) can guide antibiotic discontinuation in patients with suspected sepsis and reduce overall antibiotic use in neonates and adults [6-13]. However, data to support their use in children are limited $[6,14,15]$. We previously assessed the performance of a panel of serum biomarkers to identify critically ill children with SIRS at low-risk of bacterial infection and found that the combination of CRP and PCT, when both are low at SIRS onset, can accurately identify such children (negative predictive value $85 \%$ ) [16]. Due to the routine availability of CRP and PCT at our center, these findings served as the basis for development of a practical algorithm to guide safe discontinuation of broad-spectrum antibiotics in patients with SIRS at low risk for bacterial infection admitted to our PICU. We hypothesized that, when combined with standard clinical evaluations and examinations, measurement of biomarkers at SIRS onset could

36 • JPIDS 2020:9 (March) • Downes et al 
facilitate identification of patients at low risk of invasive bacterial infection in whom empiric antibiotics could be safely discontinued and lead to a reduction in antibiotic use. We sought to evaluate the impact of the algorithm on excess antibiotic therapy in children without proven bacterial infections.

\section{METHODS}

\section{Study Period and Setting}

We compared broad-spectrum antibiotic prescribing before and after deployment of a biomarker-based algorithm among children with new-onset SIRS without proven bacterial infections treated in the PICU at Children's Hospital of Philadelphia (CHOP) from August 10, 2012 to June 9, 2016. The CHOP PICU is a quaternary academic PICU with 55 beds and approximately 4000 annual admissions.

\section{Study Population}

Episodes were divided into 2 groups based on the date of SIRS onset in relation to implementation of the algorithm on June 10, 2015: Period 1 (August 10, 2012 to June 9, 2015) and Period 2 (June 10, 2015 to June 9, 2016). New-onset SIRS was defined as the presence of $\geq 2$ age-related criteria for SIRS within a 24-hour period [17]. Episodes were included if (1) the patient was $\leq 18$ years of age, (2) blood culture was obtained at CHOP $\leq 6$ hours of SIRS onset, and (3) a new intravenous broad-spectrum antibiotic (see definitions below) was initiated within 12 hours after blood culture collection. All new SIRS episodes $>30$ days after onset of a prior qualifying episode of SIRS were eligible. Episodes in patients with advanced directives limiting care, a history of hematopoietic stem cell or solid organ transplant, or with an absolute neutrophil count $<500$ cells/ $\mu \mathrm{L}$ were excluded because such patients were not included in the biomarker evaluation study that informed the current algorithm [16]. We also excluded patients discharged from the PICU within 2 calendar days of SIRS onset because our intervention (described below) was restricted only to the PICU, and the goal of this study was to assess the impact of the biomarker algorithm on PICU prescribing practices.

\section{Intervention}

In June 2015, CRP and PCT were incorporated into an orderset to facilitate ordering cultures, laboratory tests, and antibiotics in children with suspected sepsis in the PICU. On June 10, 2015, an algorithm was enacted stating that PICU clinicians should consider stopping antibiotics if all of the following criteria were met: (1) sterile site cultures obtained at SIRS onset revealed no growth at 48 hours, (2) both biomarkers obtained at SIRS onset were low (CRP $<4 \mathrm{mg} / \mathrm{dL}$ and PCT $<1 \mathrm{ng} / \mathrm{mL}$ ), and (3) no focal signs of infection were present on exam or by imaging. Critical care clinicians were educated about the algorithm at a division meeting in June 2015 and sent weekly reminder e-mails from July through December 2015 that contained the algorithm information. Screensavers with the algorithm were displayed on PICU workstations throughout Period 2. Supplemental Figure 1 displays the timeline of education surrounding the intervention.

Clinicians were not required to adhere to the intervention nor to utilize the orderset when placing orders for children with suspected sepsis. Measurement of biomarkers and diagnostic testing for the source of infection were performed at the discretion of the clinical team. Our Antimicrobial Stewardship Program (ASP) team, which must grant authorization for antibiotic prescriptions $>48$ hours, had access to the biomarker values in the electronic health record (EHR) but did not utilize the algorithm as part of the review process for antibiotic approval. No specific education on the algorithm was provided to ASP providers. The CHOP Institutional Review Board approved the study protocol with a waiver of informed consent/assent because this project was an evaluation of a practice change.

\section{Data Collection}

We recorded information from the EHR including patient demographics, hospital admission diagnosis, presence and category of complex chronic conditions [18], vital signs, and length of hospital and PICU stay before and after enrollment. Severity of illness at SIRS onset was assessed using the PEdiatric Logistic Organ Dysfunction-2 (PELOD-2) score [19]. Medications administered, procedures performed, and results of microbiologic cultures and imaging evaluations from 48 hours before and through 10 calendar days after enrollment were collected.

\section{Definitions}

The calendar day of SIRS onset was considered day 0 . The presence of bacterial infection was assessed by independent review of medical records (microbiology, laboratory, imaging, and clinical data) from days $0-2$ and from days 3-9 by 1 of 3 investigators (K.J.D., M.E.R., S.E.C.). Ambiguous cases were adjudicated through review by all 3 investigators. Bacterial infections were classified as present if Centers for Disease Control and Prevention (CDC) criteria for proven or probable bacterial infection were met [20], consistent with definitions used for derivation of the algorithm in our previous work [16]. A viral infection was defined as a laboratory-confirmed pathogen by polymerase chain reaction test from a clinically obtained specimen.

The primary outcome for this study was excess antibiotic use defined as administration of broad-spectrum antibiotics on days 3-9 in a patient without a proven bacterial infection ("uninfected episode"). The following antibiotics were considered broad-spectrum: aminoglycosides, third-/fourth-generation cephalosporins, carbapenems, $\beta$-lactam/ $\beta$-lactamase inhibitor combinations, daptomycin, vancomycin, and fluoroquinolones. Antibiotic exposure was defined as length of therapy ([LOT] 
number of days on which any antibiotic was administered) as well as days of therapy ([DOT] total number of antibiotics across all days) per 1000 patient-days [21]. Because patients with suspected sepsis at our institution can receive 48 hours of antibiotics without ASP approval, we calculated LOT and DOT from days 3-9 after SIRS onset for all eligible episodes; days were included through day 9 after SIRS onset, or until hospital discharge or death, whichever occurred first. If antibiotics were stopped for $>24$ hours and then restarted, antibiotics administered after stoppage were not included.

\section{Statistical Analysis}

Summary statistics were described among all eligible uninfected episodes. To compare uninfected episodes in the 2 periods, $\chi^{2}$ and Fisher's exact tests (categorical data) or Wilcoxon 2 -sample tests (continuous data) were used, as appropriate. We also compared episodes with and without bacterial infections identified on days $0-2$ irrespective of period.

Incidence rate ratios (IRRs) were calculated to compare unadjusted excess LOT and DOT per 1000 patient-days among uninfected episodes between the 2 periods. Episodes were stratified based on biomarker results: (1) both biomarkers below the cut-points ("low-risk group"), (2) either biomarker above the cut-point, or (3) both biomarkers not measured.

We then performed an interrupted time series analysis using segmented linear regression to test for a change in excess antibiotic use in the PICU after implementation of the algorithm among all uninfected episodes. The main exposure was study period (Period 2 vs 1 ). To assess the effect of time on antibiotic prescribing, calendar time was divided into 2-month blocks. Two-month blocks were chosen because the number of evaluable SIRS episodes per month was small. The primary outcome was cumulative excess LOT per 1000 patient-days per 2-month period. Secondary analyses were performed to assess excess DOT per 1000 patient-days per 2-month period.

Our base model included the following terms: bi-monthly time (2-month periods since the start of the study), study period (Period 2 vs 1 ), and number of bi-monthly periods since intervention ( 0 for all preintervention periods). The coefficient for periods since intervention reflects the change in the rate of antibiotic prescribing postintervention compared with preintervention. Meanwhile, the coefficient for study period represents differences in mean antibiotic prescribing between the periods.

Covariates were individually included in the model to determine their association with antibiotic prescribing during the study. Because of sample size considerations (ie, 23 total 2 -month periods), covariates could only be added individually to the model. First-order positive autocorrelation was detected for both the LOT (Durbin-Watson [DW] statistic = 1.35) and DOT (DW statistic $=1.25$ ) models using the Durbin-Watson statistic, so final models were adjusted for autocorrelation.
Statistical analyses were performed using SAS 9.4 (SAS Institute Inc., Cary, NC) and R version 3.3.2 (R Foundation, Vienna, Austria).

\section{RESULTS}

We identified 1378 SIRS episodes in children $\leq 18$ years of age during Period 1 and 495 in Period 2. A total of 507 (36.7\%) and 206 (41.6\%) episodes were eligible after chart review in Periods 1 and 2, respectively (Figure 1). There were 454 and 179 unique children that contributed episodes during the 2 periods. Overall, 35.9\% of reviewed episodes had a bacterial infection identified ( $\mathrm{n}=256$ of 713). The prevalence of bacterial infections was similar between periods ( $34.3 \%$ [ 174 of 507] vs $39.8 \%$ [82 of 206]; $P=.17$ ). Supplemental Table 1 compares the characteristics of episodes based on presence/absence of bacterial infection. Episodes without identified bacterial infection were from children who were younger $(P=.02)$, in the PICU longer before SIRS onset $(P<.001)$, and less often required vasopressors at SIRS onset $(P=.04)$. Pediatric intensive care unit length of stay was similar among episodes with and without bacterial infections identified $(P=.67)$.

No bacterial infection was identified in 333 and 124 SIRS episodes in Periods 1 and 2, respectively. Uninfected episodes occurred amongst similar patients in the 2 periods (Table 1), although patient races were different $(P=.05)$. In Period 1 , uninfected episodes more often involved patients with an underlying cardiovascular $(P<.001)$, respiratory $(P=.01)$, or gastrointestinal condition $(P=.05)$. Among uninfected episodes, measurement of both biomarkers at SIRS onset significantly increased from Period 1 to 2 (33.0\% [110 of 333] vs $60.5 \%$ [75 of 124]; $P<.001$ ) (Supplemental Figure 2). The frequency of Infectious Diseases consultation was similar in the 2 periods: $24.0 \%(n=80$ of 333$)$ in Period 1 compared with $19.3 \%(n=24$ of 124) in Period $2(P=.29)$.

\section{Unadjusted Analyses}

The proportion of episodes with both biomarkers below the algorithm's cut-points (low-risk group) was similar between the periods (43.6\% [ 48 of 110 ] vs $41.3 \%$ [31 of 75]; $P=.76$ ). Unadjusted excess antibiotic LOT was significantly lower in Period 2 among uninfected episodes with both biomarkers below the cut-points (IRR, 0.53; 95\% confidence interval [CI], $0.30-0.93$ ) (Table 2), as was unadjusted excess antibiotic DOT (IRR, 0.43; 95\% CI, 0.26-0.71). Meanwhile, unadjusted excess antibiotic LOT was similar among those with either biomarker above the cut-points and those in whom biomarkers were not measured, as was DOT.

\section{Time Series Analyses}

Figure 2 displays the excess LOT and DOT over time among all uninfected episodes. Supplemental Table 2 displays the

38 • JPIDS 2020:9 (March) • Downes et al 


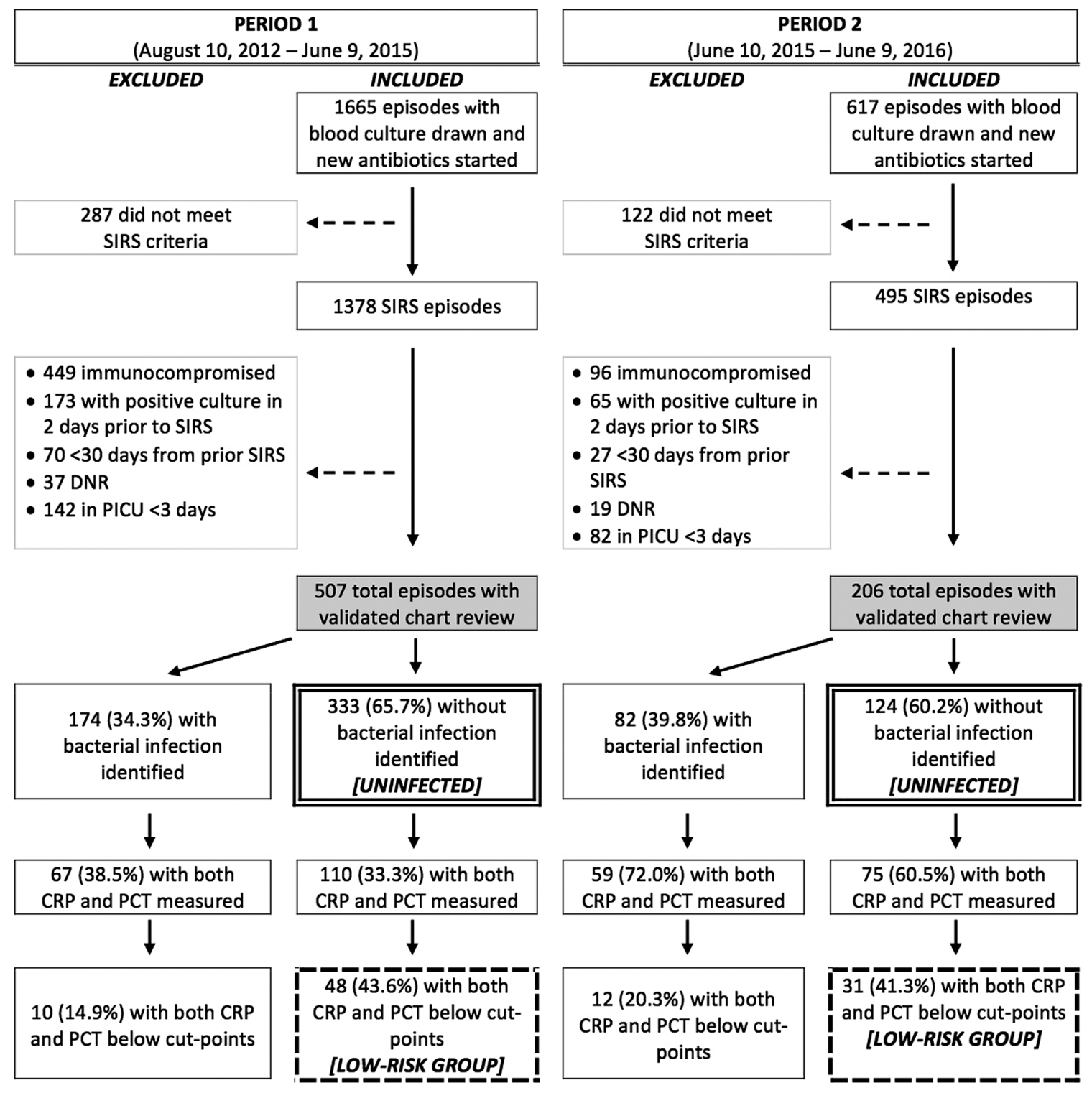

Gray boxes highlight all eligible SIRS episodes that underwent chart review to identify the presence/absence of infection.

Double-barred boxes highlight episodes without bacterial infection identified (uninfected). Uninfected episodes were the target of the intervention. Episodes in these boxes are used for primary comparisons in the manuscript, including segmented regression analyses.

Hashed boxes represent subset of uninfected episodes with both biomarkers below the algorithm's cut-points (low-risk group). Episodes in these boxes are used for comparisons of unadjusted excess length of therapy (LOT) and days of therapy (DOT) pre- and post-intervention.

Figure 1. Flow diagram of inclusion into the Optimizing Antibiotic Strategies in Sepsis (OASIS) II study. Double-barred boxes highlight episodes without bacterial infection identified (uninfected). Uninfected episodes were the target of the intervention. Episodes in these boxes are used for primary comparisons in the manuscript, including segmented regression analyses. Hashed boxes represent a subset of uninfected episodes with both biomarkers below the algorithm's cut-points (low-risk group). Episodes in these boxes are used for comparisons of unadjusted excess length of therapy and days of therapy preand postintervention. CRP, C-reactive protein; DNR, do not resuscitate; PCR, procalcitonin; PICU, pediatric intensive care unit; SIRS, systemic inflammatory response syndrome.

results of additional covariate testing in multivariable models. Segmented regression analysis showed that excess LOT among all uninfected patients in Period 2 was similar to that in Period 1
(Table 3), after adjusting for performance of surgery during the 10 days after SIRS, the most significant covariate on additional testing. There was also no difference in the slope of antibiotic 
Table 1. Patient and Clinical Characteristics of the Study Population Portion Without a Bacterial Infection During Days 0-2 of Follow-Up, by Intervention Period

\begin{tabular}{|c|c|c|c|}
\hline & Period 1 & Period 2 & \\
\hline Characteristic & $(\mathrm{n}=333)$ & $(n=124)$ & $P$ Value \\
\hline Age in years, median (IQR) & $4.1(1.2-12.3)$ & $3.8(1.5-12.6)$ & .62 \\
\hline Female sex, $\mathrm{n}(\%)$ & $158(47.5)$ & $51(41.1)$ & .23 \\
\hline Race, $\mathrm{n}(\%)$ & & & .05 \\
\hline Asian & $16(4.8)$ & $7(5.7)$ & \\
\hline Black & $125(37.5)$ & $30(24.2)$ & \\
\hline White & $118(35.4)$ & $58(46.8)$ & \\
\hline Other/Unknown & 74 (22.2) & $29(23.4)$ & \\
\hline Ethnicity, n (\%) & & & .64 \\
\hline Hispanic/Latino & $49(14.7)$ & $15(12.1)$ & \\
\hline Non-Hispanic/Latino & $283(85.0)$ & $109(87.9)$ & \\
\hline Unknown & $1(0.3)$ & $0(0)$ & \\
\hline $\begin{array}{l}\text { Presence of any complex chronic } \\
\text { condition, } \mathrm{n}(\%)\end{array}$ & $280(84.1)$ & $101(81.5)$ & .50 \\
\hline Malignancy & $28(8.4)$ & $12(9.7)$ & 67 \\
\hline Hematologic or autoimmune & 73 (21.9) & $23(18.6)$ & .43 \\
\hline Respiratory & $122(36.6)$ & $30(24.2)$ & .01 \\
\hline Gastrointestinal & $176(52.9)$ & $53(42.7)$ & .05 \\
\hline Metabolic & $77(23.1)$ & $27(21.8)$ & .76 \\
\hline Neuromuscular & $183(55.0)$ & $59(47.6)$ & .16 \\
\hline Cardiovascular & $154(46.3)$ & $35(28.2)$ & $<.001$ \\
\hline Renal & $46(13.8)$ & $12(9.7)$ & .24 \\
\hline Other congenital & $103(30.9)$ & $44(35.5)$ & .35 \\
\hline $\begin{array}{l}\text { Pre-enrollment PICU length of stay in days, } \\
\text { median (IQR) }\end{array}$ & $0(0-1)$ & $0(0-1)$ & .35 \\
\hline $\begin{array}{l}\text { Postenrollment PICU length of stay in days, } \\
\text { median (IQR) }\end{array}$ & $6(4-13)$ & $5.5(4-11.5)$ & .15 \\
\hline $\begin{array}{l}\text { Presence of positive viral panel days } 0,1 \text {, } \\
\quad \text { or } 2, \mathrm{n}(\%)\end{array}$ & $134(40.2)$ & $58(46.8)$ & .21 \\
\hline PELOD-2 score, ${ }^{a}$ median (IOR) & $6(4-9)$ & $6(4-9)$ & .54 \\
\hline Mechanical ventilation, ${ }^{\mathrm{b}} \mathrm{n}(\%)$ & $163(49.1)$ & $62(50.0)$ & .86 \\
\hline Receipt of vasopressor infusion, ${ }^{\mathrm{b}} \mathrm{n}(\%)$ & $89(26.8)$ & $28(22.6)$ & .36 \\
\hline Vasopressor use in days, ${ }^{\mathrm{b}}$ median (IQR) & $0(0-1)$ & $0(0-1)$ & .29 \\
\hline Surgery, ${ }^{b} n(\%)$ & $23(6.9)$ & $13(10.5)$ & .21 \\
\hline Chest tube in place, ${ }^{\mathrm{b}} \mathrm{n}(\%)$ & $0(0)$ & $1(0.81)$ & .27 \\
\hline Other diagnostic procedure, ${ }^{\mathrm{b}} \mathrm{n}(\%)$ & $14(4.2)$ & $7(5.7)$ & .51 \\
\hline
\end{tabular}

Abbreviations: IQR, interquartile range; PELOD-2, PEdiatric Logistic Organ Dysfunction-2; PICU, pediatric intensive care unit.

${ }^{a}$ At PICU admission.

${ }^{\mathrm{b} A t}$ any time during 10 days after enrollment. prescribing after the intervention. Excess DOT was also similar among all uninfected patients in the 2 periods (Table 3 ).

\section{Safety Analyses}

Biomarkers were below both cut-points in 12 episodes where bacterial infections were present during Period 2. All had readily identifiable infections by culture, imaging, or exam: 9 lower respiratory tract infections, 1 ventilator-associated tracheitis, 1 surgical site infection, 1 post-neurosurgical meningitis. No patients with low biomarkers had antibiotics discontinued prematurely. Of the 31 uninfected episodes in Period 2 that had low biomarkers (low-risk group), there were no identifiable bacterial infections on days 3-9 after SIRS onset requiring re-initiation of antibiotics.

\section{DISCUSSION}

We implemented a biomarker-based algorithm in our PICU to identify low-risk patients with SIRS in whom antibiotics could be safely discontinued at 48 hours. Both the unadjusted LOT and DOT decreased by approximately $50 \%$ in the subset of uninfected episodes where both biomarker values were low at SIRS onset. However, only a small fraction of uninfected episodes qualified as low-risk: $14 \%$ in Period 1 and $25 \%$ in Period 2. Thus, overall excess broad-spectrum antibiotic administration in our PICU was unchanged among all uninfected episodes. These results suggest that measurement of biomarkers can facilitate safe discontinuation of antibiotics in low-risk critically ill children with SIRS for whom bacterial infection is not clinically identified. However, future quality improvement efforts may need to focus on routine measurement of biomarkers in all children with SIRS in order to affect overall antibiotic prescribing.

When caring for critically ill children with SIRS, clinicians are often confronted with a difficult decision to determine whether a patient clinically improved because of or in spite of antibiotics. Such a decision is relatively straightforward when a bacterial infection is identified, but there is considerable uncertainty in the absence of a known infection. Biomarkers, such

Table 2. Excess Broad-Spectrum Antibiotic Prescribing in Relation to Biomarker Cut Points Among Patients Without Proven Bacterial Infections ${ }^{\mathrm{a}}$

\begin{tabular}{|c|c|c|c|c|c|c|}
\hline \multirow[b]{2}{*}{ Biomarkers } & \multicolumn{3}{|c|}{ Length of therapy (LOT) } & \multicolumn{3}{|c|}{ Days of therapy (DOT) } \\
\hline & $\begin{array}{c}\text { Period } 1 \\
\text { LOT per } 1000 \\
\text { patient-days }\end{array}$ & $\begin{array}{c}\text { Period } 2 \\
\text { LOT per } 1000 \\
\text { patient-days }\end{array}$ & $\operatorname{IRR}(95 \% \mathrm{CI})$ & $\begin{array}{c}\text { Period } 1 \\
\text { D0T per } 1000 \\
\text { patient-days }\end{array}$ & $\begin{array}{c}\text { Period } 2 \\
\text { DOT per } 1000 \\
\text { patient-days }\end{array}$ & IRR (95\% CI) \\
\hline Either above cut point & 240.8 & 272.7 & $1.13(0.80-1.60)$ & 371.2 & 450.0 & $1.21(0.92-1.59)$ \\
\hline Both below cut points (low-risk) & 196.8 & 103.9 & $0.53(0.30-0.93)$ & 301.2 & 129.9 & $0.43(0.26-0.71)$ \\
\hline Biomarkers not measured & 238.6 & 214.6 & $0.90(0.67-1.21)$ & 385.8 & 323.9 & $0.84(0.66-1.07)$ \\
\hline All episodes without proven bacterial infection & 232.6 & 207.7 & $0.89(0.73-1.09)$ & 370.1 & 320.5 & $0.87(0.71-1.06)$ \\
\hline
\end{tabular}

Abbreviations: DOT, days of therapy; LOT, length of therapy.

aLow-risk cut-points defined as C-reactive protein $<4 \mathrm{mg} / \mathrm{dL}$ and procalcitonin $<1 \mathrm{ng} / \mathrm{mL}$. 

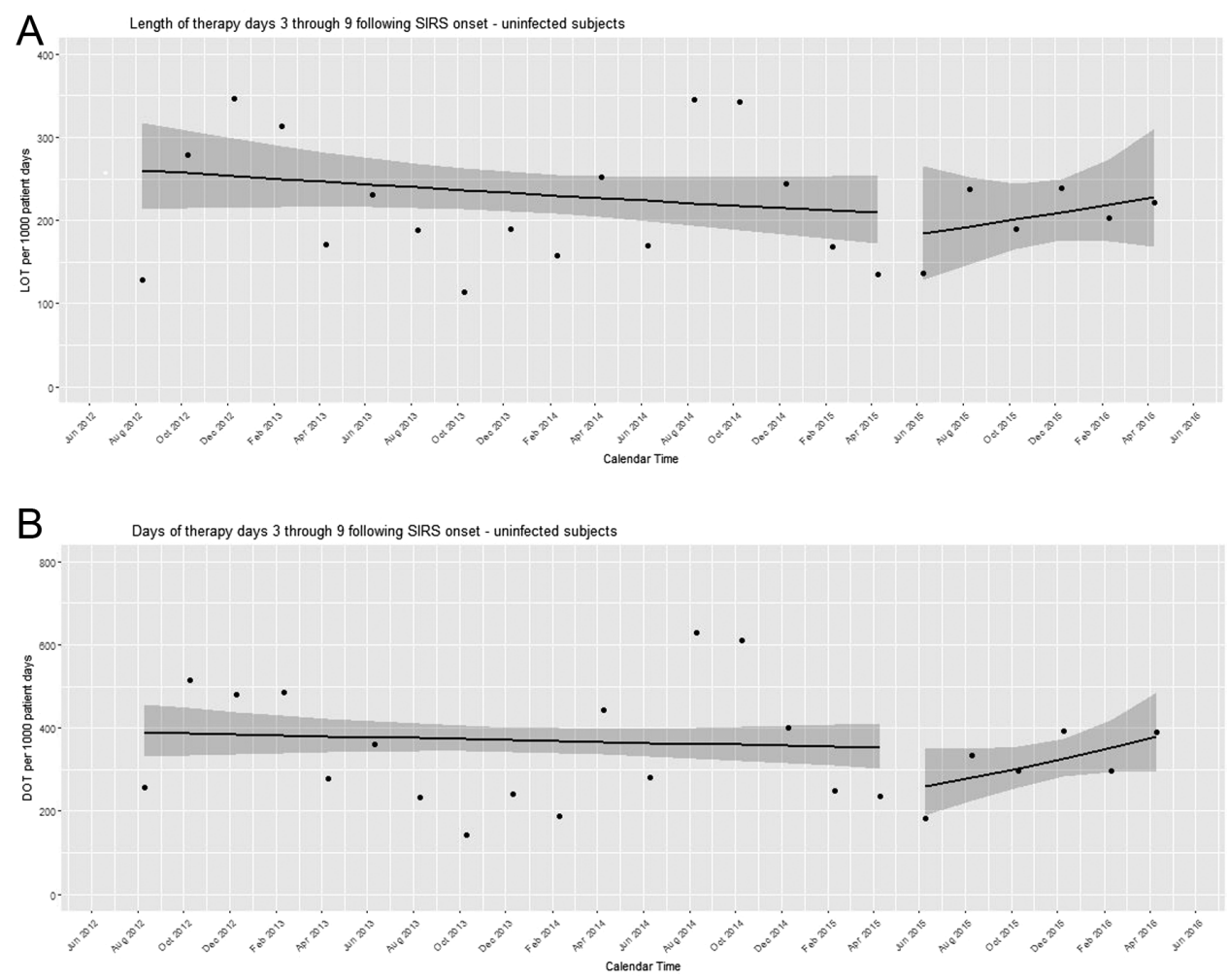

Figure 2. Excess broad-spectrum antibiotic administration among uninfected episodes. Excess length of therapy (A) and days of therapy (B) on days 3 through 9 in uninfected episodes. LOT, length of therapy; SIRS, systemic inflammatory response syndrome.

as CRP and PCT, when measured at SIRS onset, may provide support that the cause of illness is unlikely to be bacterial and recovery occurred in spite of antibiotics. Thus, reassuring biomarkers may offer objective data to substantiate a decision to stop antibiotics. The fact that no patients classified as low-risk in Period 2 had untoward infectious complications after discontinuation of antibiotics supports this idea, although the number was small. Although both PCT and CRP outperform traditional

Table 3. Multivariable Linear Regression for Assessment of Excess Broad-Spectrum Antibiotic Administration (Days 3-9) Among Uninfected Patients

\begin{tabular}{lcc}
\hline Length of Therapy & & \\
\hline Covariate & Coefficient & PValue \\
\hline Period 2 vs Period 1 & 0.9 & .99 \\
Bimonthly time & -6.0 & .18 \\
Time since intervention ${ }^{\mathrm{a}}$ & 10.8 & .55 \\
Surgery (Y/N) & 554.7 & .01 \\
Days of Therapy & & \\
Period 2 vs Period 1 & -40.6 & .76 \\
Bimonthly time & -10.9 & .19 \\
Time since intervention ${ }^{\mathrm{a}}$ & 29.4 & .37 \\
Surgery (Y/N) & 1144.0 & .003 \\
\hline
\end{tabular}

Abbreviations: N, no; Y, yes.

${ }^{a}$ Coefficient of time since intervention reflects the difference in the slopes of antibiotic prescribing between study periods markers of infection in differentiating bacterial from viral infections and sterile inflammation [22], no biomarkers have perfect sensitivity and specificity. Therefore, biomarkers can only supplement and not overrule clinical judgment when it comes to antibiotic decisions.

Culture-negative sepsis may occur for a variety of reasons, including incomplete infectious evaluation or pretreatment with antibiotics, and the adequacy of the infectious evaluation must be considered in the decision to stop antibiotics. Approximately half of severe sepsis hospitalizations are attributable to culture-negative sepsis, a diagnosis that is associated with more medically complex patients and higher mortality $[23,24]$. However, there is evidence that clinicians can be more aggressive in de-escalating broad-spectrum antibiotics in culture-negative sepsis, and that appropriate de-escalation may not worsen outcomes [23]. It is important to consider the need for continuing antibiotic therapy on a case-by-case basis and to develop an appropriate monitoring plan if antibiotic therapy is stopped or de-escalated.

Reducing unnecessary antibiotic use is an important goal of pediatric ASPs, but defining antibiotic use as unnecessary is difficult in the critical care setting. To be conservative, we defined excess antibiotics as those administered 3 or more calendar days after SIRS onset in a child without evidence of a bacterial infection. Because our institution's ASP requires 
approval for extension of broad-spectrum antibiotics beyond 48 hours, we allowed PICU clinicians to make antibiotic decisions per standard practice before categorizing use as unnecessary. Children who were discharged from the PICU before day 3 were not analyzed, and this occurred more often during Period 2. It is possible that our algorithm helped identify these lowest-risk patients more rapidly, reducing the measurable impact of our intervention.

After implementation of our algorithm, LOT was curtailed by approximately $50 \%$ in the subset of uninfected patients with low biomarkers. The lack of a significant decrease in antibiotic LOT overall among uninfected patients might have been due to the small number of patients to which our low-risk definition applied and slow uptake of routine biomarker measurement by clinicians. Even in Period 2, less than two-thirds of children had biomarkers measured and, of uninfected children that had biomarkers measured, only 31 (40\%) had low CRP and PCT. Therefore, only 25\% of all uninfected episodes in Period 2 were categorized as low-risk. With increased collection of biomarkers over time, we may have seen a significant reduction in overall excess antibiotic administration had we used a longer postintervention period.

There are limitations to our study. First, this was a passive intervention on antibiotic prescribing in our PICU. We intentionally selected an algorithm that could be easily implemented in other centers; however, the use of more formal quality improvement methods may have improved adherence and further reduced unnecessary antibiotic administration. Second, a large number of SIRS episodes were excluded from our analyses (62\%). Because we excluded episodes in children with underlying conditions that inherently affect antibiotic decisions (immunocompromised, do not resuscitate), our findings are not generalizable to all critically ill children. Studies suggest that the kinetics of PCT and CRP in neutropenic children may be similar to nonneutropenic children [25]. However, decisions around antibiotic cessation differ from those in other children. Third, calls and recommendations are not formally tracked by our ASP, and, therefore, we cannot determine whether our algorithm indirectly influenced ASP guidance during Period 2. Finally, we used CDC surveillance definitions to characterize infections, which risks potential misclassification bias. Although these definitions provide a structured approach for infection identification and classification, they do not always align with clinicians' real-time diagnoses. All medical records were manually reviewed for the presence or absence of infection, and cases that could not be classified clearly were adjudicated by 3 team members. Future studies need to evaluate the cost-effectiveness of universal biomarker measurement in children with SIRS to fully understand their impact, although adult studies suggest that they are cost effective $[9,26]$.

\section{CONCLUSIONS}

In conclusion, after implementation of a biomarker-based algorithm, overall antibiotic prescribing was unchanged among uninfected children in our PICU. However, antibiotic use was reduced in the subset of patients with absence of clinically identified bacterial infection and low CRP and PCT values at SIRS onset. Measurement of CRP and PCT may supplement standard diagnostic tests and clinical evaluations to help to reduce unnecessary antibiotic therapy in this subset of lowest-risk patients.

\section{Supplementary Data}

Supplementary materials are available at Journal of the Pediatric Infectious Diseases Society online.

\section{Acknowledgments}

Disclaimer. The funding agencies had no role in the design and conduct of the study, collection, management, analysis, and interpretation of the data, or preparation, review, or approval of the manuscript.

Financial support. This work was supported by the CDC Cooperative Agreement FOA CK11-001-Epicenters for the Prevention of Healthcare Associated Infections (to E. L.). K. J. D. is supported by the Eunice Kennedy Shriver National Institute Of Child Health and Human Development of the National Institutes of Health under Award Number K23HD091365. F. B. received career development support from the Eunice Kennedy Shriver National Institute of Child Health and Human Development (Grant 1K23HD082368).

Potential conflicts of interest. K. J. D. has received research support from Merck, Inc. and Pfizer, Inc. unrelated to the current work. All authors have submitted the ICMJE Form for Disclosure of Potential Conflicts of Interest. Conflicts that the editors consider relevant to the content of the manuscript have been disclosed.

\section{References}

1. Weiss SL, Fitzgerald JC, Balamuth F, et al. Delayed antimicrobial therapy increases mortality and organ dysfunction duration in pediatric sepsis. Crit Care Med 2014; 42:2409-17.

2. Kumar A, Roberts D, Wood KE, et al. Duration of hypotension before initiation of effective antimicrobial therapy is the critical determinant of survival in human septic shock. Crit Care Med 2006; 34:1589-96.

3. Centers for Disease Control and Prevention. Antibiotic resistance threats in the United States. 2013. Available at: https://www.cdc.gov/drugresistance/pdf/ ar-threats-2013-508.pdf. Accessed March 13, 2015

4. Grohskopf LA, Huskins WC, Sinkowitz-Cochran RL, et al. Use of antimicrobial agents in United States neonatal and pediatric intensive care patients. Pediatr Infect Dis J 2005; 24:766-73.

5. Blinova E, Lau E, Bitnun A, et al. Point prevalence survey of antimicrobial utilization in the cardiac and pediatric critical care unit. Pediatr Crit Care Med 2013; 14:e280-8.

6. Stocker M, Fontana M, El Helou S, et al. Use of procalcitonin-guided decision-making to shorten antibiotic therapy in suspected neonatal early-onset sepsis: prospective randomized intervention trial. Neonatology 2010; 97:165-74.

7. Bouadma L, Luyt CE, Tubach F, et al. Use of procalcitonin to reduce patients' exposure to antibiotics in intensive care units (PRORATA trial): a multicentre randomised controlled trial. Lancet 2010; 375:463-74.

8. Hohn A, Schroeder S, Gehrt A, et al. Procalcitonin-guided algorithm to reduce length of antibiotic therapy in patients with severe sepsis and septic shock. BMC Infect Dis 2013; 13:158.

9. Westwood M, Ramaekers B, Whiting P, et al. Procalcitonin testing to guide antibiotic therapy for the treatment of sepsis in intensive care settings and for suspected bacterial infection in emergency department settings: a systematic review and cost-effectiveness analysis. Health Technol Assess 2015; 19:V-XXV, 1-236.

10. Wilke MH, Grube RF, Bodmann KF. The use of a standardized PCT-algorithm reduces costs in intensive care in septic patients - a DRG-based simulation model. Eur J Med Res 2011; 16:543-8. 
11. Schroeder S, Hochreiter M, Koehler T, et al. Procalcitonin (PCT)-guided algorithm reduces length of antibiotic treatment in surgical intensive care patients with severe sepsis: results of a prospective randomized study. Langenbecks Arch Surg 2009; 394:221-6.

12. Kopterides P, Siempos II, Tsangaris I, et al. Procalcitonin-guided algorithms of antibiotic therapy in the intensive care unit: a systematic review and meta-analysis of randomized controlled trials. Crit Care Med 2010; 38:2229-41.

13. Albrich WC, Harbarth S. Pros and cons of using biomarkers versus clinical decisions in start and stop decisions for antibiotics in the critical care setting. Intensive Care Med 2015; 41:1739-51.

14. Baer G, Baumann P, Buettcher M, et al. Procalcitonin guidance to reduce antibiotic treatment of lower respiratory tract infection in children and adolescents (ProPAED): a randomized controlled trial. PLoS One 2013; 8:e68419.

15. Ross RK, Keele L, Kubis S, et al. Effect of the procalcitonin assay on antibiotic use in critically ill children. J Pediatric Infect Dis Soc 2018; 7:e43-6.

16. Downes KJ, Weiss SL, Gerber JS, et al. A pragmatic biomarker-driven algorithm to guide antibiotic use in the pediatric intensive care unit: the optimizing antibiotic strategies in sepsis (OASIS) study. J Pediatric Infect Dis Soc 2017; 6:134-41.

17. Goldstein B, Giroir B, Randolph A. International pediatric sepsis consensus conference: definitions for sepsis and organ dysfunction in pediatrics. Pediatr Crit Care Med 2005; 6:2-8.

18. Feudtner C, Hays RM, Haynes G, et al. Deaths attributed to pediatric complex chronic conditions: national trends and implications for supportive care services. Pediatrics 2001; 107:E99.
19. Leteurtre S, Duhamel A, Salleron J, et al. PELOD-2: an update of the PEdiatric logistic organ dysfunction score. Crit Care Med 2013; 41:1761-73.

20. Horan TC, Andrus M, Dudeck MA. CDC/NHSN surveillance definition of health care-associated infection and criteria for specific types of infections in the acute care setting. Am J Infect Control 2008; 36:309-32.

21. Morris AM. Antimicrobial stewardship programs: appropriate measures and metrics to study their impact. Curr Treat Options Infect Dis 2014; 6:101-12.

22. Lautz AJ, Dziorny AC, Denson AR, et al. Value of procalcitonin measurement for early evidence of severe bacterial infections in the pediatric intensive care unit. J Pediatr 2016; 179:74-81.e2.

23. Moraes RB, Guillén JA, Zabaleta WJ, Borges FK. De-escalation, adequacy of antibiotic therapy and culture positivity in septic patients: an observational study. Rev Bras Ter Intensiva 2016; 28:315-22.

24. Gupta S, Sakhuja A, Kumar G, et al. Culture-negative severe sepsis: nationwide trends and outcomes. Chest 2016; 150:1251-9.

25. de Araujo OR, Salomão R, Brunialti MC, et al. Cytokine kinetics in febrile neutropenic children: insights on the usefulness as sepsis biomarkers, influence of filgrastim, and behavior of the IL-23/IL-17 pathway. Mediators Inflamm 2017; 2017:8291316.

26. Kip MM, Kusters R, IJzerman MJ, Steuten LM. A PCT algorithm for discontinuation of antibiotic therapy is a cost-effective way to reduce antibiotic exposure in adult intensive care patients with sepsis. J Med Econ 2015; 18:944-53. 